\title{
Escala de Satisfacción Familiar por Adjetivos (ESFA) en escolares y adolescentes mexicanos: datos normativos
}

\author{
Gabriela Tercero Quintanilla, ${ }^{1}$ María del Pilar Deyanira Lavielle Sotomayor, ${ }^{2}$ \\ Onofre Muñoz Hernández, ${ }^{3}$ Patricia Clark Peralta, ${ }^{4}$ Mara Medeiros Domingo, ${ }^{5}$ \\ Alejandra Hernández Roque, ${ }^{6}$ Mercedes Luque Coqui ${ }^{6}$
}

Artículo original

\section{SUMMARY}

\section{Introduction}

Familiar satisfaction results from the continuous interplay of verbal and/or physical interactions between subjects and the other members of their family. This satisfaction maintain relations to the coherence, fairness, fun, support, lack of conflicts, affective proximity, confidence, cohesion, adaptation, allocation and acceptance of roles and tasks, open communication and the acceptance in general of the own family. The use of developed psychological tests constructed in other countries is a frequent practice in the world. The validation and standardization of the test imply to establish measurement rules and scales of qualification for a determined population, when it is different from the original population in which it was created or where the instrument is used habitually.

The objective of this study was to collect the normative data of the Family Satisfaction by Adjectives Scale (FSAS) in children and adolescents of Mexico City.

\section{Material and methods}

A cross-sectional study was performed in 476 men and women; 8 to 15 years 11 months of age. The survey family satisfaction by adjectives scale (FSAS) by Barraca and López-Yarto was applied. The data were analyzed with the Statistical Package for Social Sciences (SPSS v. 17).

\section{Results}

The sample was conformed by 226 men $(47.5 \%)$ and 250 women $(52.5 \%)$. Of these, 237 were children (8 to 11 years and 11 months of age) and 239 were adolescents ( 12 to 15 years and 11 months of age). It was found that Family Satisfaction by Adjectives Scale (FSAS) has very good reliability $(\alpha=.89)$, construct ( $50 \%$ total variance), content and discriminative validity among children and adolescents (p.000).

Key words: Family Satisfaction by Adjectives Scale (FSAS), test validation, test validity, test reliability, normative data.

\section{RESUMEN}

\section{Introducción}

La satisfacción familiar es el resultado del continuo juego de interacciones verbales $\mathrm{y} / \mathrm{o}$ físicas que mantiene un sujeto con los otros miembros de su familia y guarda relación con la coherencia, la equidad en el hogar, la diversión, el apoyo, la falta de conflictos, la cercanía afectiva, la confianza, la cohesión, la adaptación, la asignación y aceptación de roles y tareas, la comunicación abierta y la aceptación en general de la propia familia. La utilización de pruebas psicológicas construidas en otros países es una práctica frecuente no sólo en nuestro país sino en todo el mundo. La validación y estandarización de las mismas implica establecer reglas de medición y escalas de calificación para una población determinada, cuando es diferente de la población original en la que se creó o se utiliza habitualmente el instrumento.

El objetivo de este estudio fue obtener los datos normativos de la Escala de Satisfacción Familiar por Adjetivos (ESFA) en escolares y adolescentes mexicanos de la Ciudad de México.

\section{Material y métodos}

Se realizó una encuesta transversal a 476 hombres y mujeres, de 8 a 15 años 11 meses, a quienes se les aplicó la escala de satisfacción familiar por adjetivos (ESFA) de Barraca y López-Yarto. Los datos se analizaron con el Paquete Estadístico para las Ciencias Sociales (SPSS v. 17).

\section{Resultados}

La muestra se conformó por 226 hombres (47.5\%) y 250 mujeres (52.5\%). De éstos, 237 fueron escolares de 8 a 11 años 11 meses y 239 adolescentes de 12 a 15 años 11 meses. Se encontró que la escala posee muy buena confiabilidad $(\alpha=.89)$, validez de constructo (varianza total de 50\%), de contenido y discriminante entre escolares y adolescentes (p.000).

Palabras clave: Escala de Satisfacción Familiar por Adjetivos (ESFA), validación, validez, confiabilidad, datos normativos.

\footnotetext{
Departamento de Psiquiatría y Medicina del Adolescente. Hospital Infantil de México Federico Gómez.

Unidad de Investigación en Epidemiología Clínica. Hospital de Especialidades. Centro Médico Nacional Siglo XXI. IMSS.

Dirección de Investigación. Hospital Infantil de México Federico Gómez.

4 Unidad de Epidemiología Clínica. Hospital Infantil de México Federico Gómez.

Laboratorio de Investigación en Nefrología. Hospital Infantil de México Federico Gómez.

Departamento de Psiquiatría y Medicina del Adolescente. Hospital Infantil de México Federico Gómez.
}

Correspondencia: Men C. Gabriela Tercero Quintanilla. Departamento de Psiquiatría y Medicina del Adolescente. Hospital Infantil de México Federico Gómez. Dr. Márquez 162, Col. Doctores, Deleg. Cuauhtémoc. 06720, México DF. Tel: 5228 - 9917, ext. 2038. E-mal: terceroq@yahoo.com

Recibido primera versión: 14 de agosto de 2011. Segunda versión: 9 de abril de 2013. Aceptado: 8 de mayo de 2013. 


\section{INTRODUCCIÓN}

La investigación sobre la familia ha sido abordada por diferentes disciplinas científicas como la sociología, la antropología, la psicología, la medicina y la pedagogía. Cada una ha intentado desde su campo analizar y estudiar los aspectos positivos y negativos que influyen en el proceso de desarrollo de cada uno de sus miembros. ${ }^{1}$

La evaluación de la satisfacción familiar se inicia en la década de 1970 en el ámbito de la psicología y sociología. Sin embargo, son pocos los instrumentos que evalúan el funcionamiento familiar que estén respaldados por modelos teóricos consolidados y pocos los esfuerzos que se han hecho en psicología por definir el constructo como un aspecto fundamental de las relaciones familiares. ${ }^{1-3}$

Con respecto al funcionamiento familiar, Olson, Portner y Lavee ${ }^{2}$ desarrollaron en Estados Unidos en 1978 las Family Adaptability and Cohesion Evaluation Scales (FACES), que tienen por objetivo medir la satisfacción familiar con respecto a la familia real y la ideal. Sin embargo, debido a que no se pudo obtener su validez empírica, en 1982 Olson y Wilson² publicaron la escala de satisfacción familiar (Family Satisfaction Scale), que es uno de los instrumentos más utilizados para estudiar el bienestar y el ajuste familiar en dos dimensiones; la cohesión y la capacidad de adaptación de la familia a diversas circunstancias. ${ }^{2}$ La confiabilidad alcanzada mediante el alfa de Cronbach es de .92 y la varianza total explicada en un solo factor es de $57.9 \%{ }^{4}$

Otro instrumento usado para evaluar el funcionamiento familiar es la Escala de Satisfacción con la Vida Familiar de Kansas (Kansas Family Life Satisfaction Scale, KFLS) de Schumm et al. (1986). Este instrumento fue diseñado para evaluar la satisfacción de las relaciones familiares de padres, de padres a hijos (al menos dos) y entre hermanos. ${ }^{2}$

La Escala de Satisfacción Familiar (Family Satisfaction Scale) de Carver y Jones (1992) es un instrumento que se responde en una escala tipo Likert y que evalúa la satisfacción del individuo con su familia de origen. Los autores reportaron aceptables niveles de confiabilidad (coeficiente alfa de Cronbach .95) y estabilidad temporal de las puntuaciones. ${ }^{2}$

Debido a que casi todos los autores consideran que la satisfacción familiar es un juicio cognitivo, es decir, una valoración que realiza el sujeto de diferentes aspectos (no explícitos) de su vida familiar real comparada con una ideal, surge la Escala de Satisfacción Familiar por Adjetivos (ESFA) de Barraca y López-Yarto (1996). ${ }^{2}$

La satisfacción familiar es el resultado del continuo juego de interacciones (verbales y/o físicas) que mantiene un sujeto con los otros miembros de su familia. Es la suma de distintos sentimientos que se despiertan en el sujeto al estar con su familia, por lo que cada miembro del grupo puede tener una satisfacción completamente diferente; ${ }^{2,3}$ es decir, la satisfacción familiar es una valoración del estado de ánimo despertado por la familia basada en el número de experiencias positivas y negativas vividas en ella. ${ }^{5}$
Cuando las interacciones son positivas para el sujeto, tenderá a estar satisfecho y formulará un juicio satisfactorio de su familia; mientras que cuando son negativas, tenderá a estar insatisfecho. ${ }^{2,5}$

Esta condición de insatisfacción se ha asociado a ambientes familiares con elevados niveles de frustración, resentimiento, agresión y enojo, menos cohesión y más conflictos, dificultades en la comunicación (ya que es poco clara y confusa), aislamiento, escasas redes de apoyo; utilización de estrategias de afrontamiento disfuncionales para intentar resolver los problemas que se les presentan,;,6-8 ambigüedad en los límites; es decir, en muchas ocasiones los miembros de la familia no saben cómo relacionarse unos con otros y cuáles son sus roles y tareas. 2,6,9 Esto tiene como consecuencia que el individuo no se siente integrado a su familia. ${ }^{2,10}$

La utilización de pruebas psicológicas construidas en otros países es una práctica frecuente no sólo en nuestro país sino en todo el mundo. Sin embargo, el uso de un instrumento en un contexto cultural diferente al que fue creado puede afectar la eficacia de la evaluación y producir resultados erróneos. ${ }^{11-14}$

La validación y estandarización de las pruebas psicológicas implica establecer reglas de medición y escalas de calificación para una población determinada. ${ }^{13,15}$ La adaptación del instrumento resulta necesaria cuando la población objetivo difiere de la población original en la que se creó o se utiliza habitualmente ${ }^{16}$ e implica la transformación, adición o eliminación de algunos reactivos de la escala original, ya que pueden cambiar su significado en cuanto a características como cultura, país o lengua. ${ }^{11-13}$

El principal criterio para describir las propiedades psicométricas de cualquier prueba (es decir, sus características matemáticas y estadísticas) son la confiabilidad y validez. ${ }^{11,15}$

La confiabilidad se refiere a la consistencia de las puntuaciones obtenidas por los mismos individuos cuando son examinados con la misma prueba en diferentes ocasiones. ${ }^{15,17,18}$ Se determina por medio de un coeficiente de correlación cuyo valor va de 0 a 1 . Cuanto más se acerque el coeficiente a 1, más confiable es la prueba. ${ }^{17,18}$

Los procedimientos para obtener la confiabilidad de un instrumento son el test-retest, formas paralelas, división por mitades, la consistencia interna (coeficiente alfa de Cronbach o el de Kuder-Richardson) y entre evaluadores. ${ }^{13,17-19}$

La validez, es decir, el grado de exactitud con el que una prueba mide efectivamente lo que dice medir en una población determinada y en las condiciones normales de aplicación, puede ser de contenido, predictiva, en relación con un criterio o de constructo. ${ }^{11,13,15,17-19}$

Cuando las condiciones de validación y estandarización de la prueba están bien definidas y su utilización es idéntica en todos los sujetos examinados, el siguiente paso es la interpretación de las puntuaciones logradas por los sujetos evaluados. ${ }^{15,17-19}$ Esta interpretación se realiza comparando la calificación obtenida por el sujeto con las puntuaciones 
contenidas en los cuadros de normas o baremos. ${ }^{17-20}$ Las formas para obtener estas normas son: baremos cronológicos, percentiles (puntaje derivado que transforma la calificación directa en una escala del 1 al 100) y puntajes típicos (aquellos que tienen como unidad a fracciones de la desviación estándar como los estándares o los normalizados). 17,18,20

El objetivo de este estudio fue obtener los datos normativos (centiles) de la Escala de Satisfacción Familiar por Adjetivos (ESFA) en escolares y adolescentes mexicanos de la Ciudad de México.

\section{MATERIAL Y MÉTODOS}

Se realizó una encuesta transversal a 476 hombres y mujeres de ocho a 15 años 11 meses. La muestra se dividió en dos grupos: 237 escolares (alumnos de $3^{\circ}$ a $6^{\circ}$ de primaria) y 239 adolescentes (alumnos de $1^{\circ}$ a $3^{\circ}$ de secundaria), ambas públicas. Los centros educativos se seleccionaron porque se encontraban en un área circundante al Hospital Infantil de México Federico Gómez y de acuerdo con las facilidades que brindaron para la realización del estudio. Los cuestionarios fueron aplicados por psicólogos calificados que supervisaron la forma de responder y resolvieron a los sujetos sus dudas con respecto al significado de los antónimos.

La Escala de Satisfacción Familiar por Adjetivos (ESFA) de Barraca y López-Yarto (1996) ${ }^{2}$ es un instrumento breve y sencillo diseñado en España para evaluar la satisfacción familiar expresada por los sujetos por medio de distintos adjetivos. Constituye una medida de la percepción global que tiene el sujeto sobre su situación familiar.

Está compuesta por 27 reactivos, cada uno formado por una pareja de adjetivos antónimos que tratan de evocar respuestas afectivas en el sujeto y que tienen su origen en las interacciones verbales y/o físicas que se producen entre él y los demás miembros de su familia. Puede ser aplicada tanto individual como colectivamente a partir de los 16 años y sirve para fines clínicos, de investigación y en otros campos de la psicología y pedagogía donde sea necesario o conveniente el estudio de la situación familiar (peritajes, juicios, etc.). Por su sencillez, no lleva más de 10 minutos completarla.

Las posibles puntuaciones de cada uno de los antónimos es de 1 (aspecto negativo) a 6 (aspecto positivo), llamadas puntuaciones directas, que se suman para obtener la puntuación total de la escala (máximo 162 puntos). Con la puntuación total se obtienen los centiles según los cuales se determina la satisfacción familiar: 10-20 muy baja; 21-39 baja; 40-60 media; 61-79 alta y 80-90 muy alta satisfacción familiar; sin embargo, en general las puntuaciones por encima del centil cincuenta indican una vivencia satisfactoria de la propia familia.

Cuanta más alta es la puntuación, puede entenderse que las interacciones con la familia resultan más gratificantes y que el sujeto valora sus relaciones familiares positivas, agradables, de apoyo, tranquilidad, bienestar, respeto y comprensión entre sus miembros.

La consistencia interna de la ESFA fue obtenida por el alfa de Cronbach ( $\alpha=.97$ para la muestra total) y por el método de mitades partidas ( $\alpha=.96$ para la muestra total). La estabilidad temporal se obtuvo por el test-retest a las cuatro semanas con un coeficiente de correlación de .75.

La validez de constructo se comprobó mediante un análisis factorial de componentes principales y el criterio de Kaiser para la apreciación de los factores. Se obtuvieron tres factores con valores eigen mayores a uno que explican el $70.8 \%$ de la varianza; sin embargo, debido a que el primer factor por sí solo explica el 62.3\%, Barraca y López-Yarto ${ }^{1}$ consideraron que la escala tiene una sola dimensión.

Para estimar la validez de criterio de la ESFA, se escogieron dos escalas: la Escala de Satisfacción Familiar de Olson y Wilson $(r=.79)$ y la Escala de Satisfacción Familiar de Carver y Jones $(r=.65)$. Asimismo se obtuvo una validez discriminante que permite distinguir entre una muestra general y un grupo clínico en terapia familiar ( $\mathrm{U}=68.5$; p.003). ${ }^{2}$

Para obtener los datos normativos (baremos) de la ESFA en escolares y adolescentes, se utilizó el Paquete Estadístico para las Ciencias Sociales (SPSS v.17).

Se obtuvieron las medidas de tendencia central y de dispersión de las variables de sexo y grupo de edad. Se utilizó la $\chi^{2}$ para determinar si había diferencias estadísticamente significativas en las variables con respecto a la puntuación total obtenida por los sujetos en la escala. La determinación de las propiedades psicométricas de la ESFA se realizó con el análisis factorial para la validez y se obtuvieron los coeficientes de confiabilidad alfa de Cronbach y mitades partidas. Finalmente se obtuvieron los percentiles de la satisfacción familiar para escolares y adolescentes.

\section{RESULTADOS}

Se aplicaron en total 538 cuestionarios, sin embargo, para el análisis se eliminaron 62 (12\%) porque no fueron respondidos correctamente por los menores.

Para los 476 sujetos reportados, se consideraron dos determinantes: sexo (hombre o mujer) y grupo de edad (escolar o adolescente). Se encontró que las dos variables poseen una distribución libre, por lo que se reportan las medidas de tendencia central y de dispersión para datos no paramétricos.

Sexo: La muestra estuvo conformada por 226 hombres (47.5\%) y 250 mujeres (52.5\%). El valor de $\chi^{2}$ obtenido para determinar si existen diferencias entre hombres y mujeres con respecto a la puntuación total obtenida en el cuestionario no fue estadísticamente significativo $(\mathrm{p}=0.51)$.

Grupo de edad: Se tomaron en cuenta 237 escolares de ocho a 11 años 11 meses (49.8\%) y 239 adolescentes de 12 a 15 años 11 meses (50.2\%). La mediana de edad de la muestra total fue 11 años 11 meses; 10 años 6 meses para el grupo 
Cuadro 1. Propiedades psicométricas de la ESFA

\begin{tabular}{lccc}
\hline & \multicolumn{3}{c}{ Valor } \\
\cline { 2 - 4 } & $\begin{array}{c}\text { Muestra total } \\
\mathrm{N}=476\end{array}$ & $\begin{array}{c}\text { Escolares } \\
\mathrm{N}=237\end{array}$ & $\begin{array}{c}\text { Adolescentes } \\
\mathrm{N}=239\end{array}$ \\
\hline Tamaño muestra & .91 & .83 & .90 \\
Varianza & $50 \%$ & $58 \%$ & $57 \%$ \\
Número de factores & 6 & 8 & 6 \\
Confiabilidad: & & & \\
- Alfa Cronbach & .89 & .86 & .91 \\
- Mitades partidas & .85 & .80 & .87 \\
\hline
\end{tabular}

Cuadro 2. Datos del análisis factorial de la ESFA en escolares y adolescentes

\begin{tabular}{cccc}
\hline Factor & Valor Eigen & \% Varianza & $\begin{array}{r}\text { \% Varianza } \\
\text { acumulada }\end{array}$ \\
\hline I & 7.265 & 26.906 & 29.906 \\
II & 1.626 & 6.022 & 32.954 \\
III & 1.299 & 4.813 & 37.766 \\
IV & 1.138 & 4.217 & 41.969 \\
V & 1.060 & 3.926 & 45.895 \\
VI & 1.031 & 3.818 & 49.708 \\
\hline
\end{tabular}

Cuadro 3. Factores de la ESFA en escolares y adolescentes

\begin{tabular}{cl}
\hline Factor & Antónimos \\
\hline I & $13,15,16,18,19,21,23,24,25,27$ \\
II & $1,2,3,4,5,7,8,12$ \\
III & $9,17,20$ \\
IV & $6,22,26$ \\
V & 10,14 \\
VI & 11 \\
\hline
\end{tabular}

Cuadro 4. Consistencia interna de la ESFA

\begin{tabular}{|c|c|c|c|c|c|}
\hline Antónimo & Valor $r^{*}$ & Antónimo & Valor $r^{*}$ & Antónimo & Valor $r^{*}$ \\
\hline 1 & .884 & 10 & .889 & 19 & .884 \\
\hline 2 & .883 & 11 & .890 & 20 & .883 \\
\hline 3 & .884 & 12 & .883 & 21 & .882 \\
\hline 4 & .884 & 13 & .883 & 22 & .885 \\
\hline 5 & .883 & 14 & .884 & 23 & .885 \\
\hline 6 & .890 & 15 & .883 & 24 & .883 \\
\hline 7 & .884 & 16 & .883 & 25 & .882 \\
\hline 8 & .885 & 17 & .883 & 26 & .883 \\
\hline 9 & .884 & 18 & .882 & 27 & .883 \\
\hline
\end{tabular}

$r^{*}$ Coeficiente alfa de Cronbach.

de escolares y 14 para el grupo de adolescentes. Hubo diferencias estadísticamente significativas en la mediana de puntuación total obtenida en la escala por grupo de edad $\left(\chi^{2}=108.491 ; \mathrm{p}=0.002\right)$, siendo 140 puntos para escolares $\mathrm{y}$ 134 para adolescentes.

Para estimar el tamaño de la muestra necesario para obtener los datos normativos, se consideró el índice KMO (Kaiser-Meyer-Olkin) sobre suficiencia de la muestra que fue para la muestra total de .92; para el grupo de escolares,
Cuadro 5. Percentiles de la satisfacción familiar (SF) para escolares y adolescentes

\begin{tabular}{lccc}
\hline Percentil & Interpretación SF & $\begin{array}{c}\text { Valor } \\
\text { escolares }\end{array}$ & $\begin{array}{c}\text { Valor } \\
\text { adolescentes }\end{array}$ \\
\hline 10 & Muy baja & $<114$ & $<105$ \\
20 & Muy baja & 123 & 115 \\
30 & Baja & 130 & 125 \\
40 & Media & 136 & 129 \\
50 & Media & 140 & 134 \\
60 & Media & 144 & 138 \\
70 & Alta & 148 & 144 \\
80 & Muy alta & 151 & 148 \\
90 & Muy alta & $155>$ & $154>$ \\
\hline
\end{tabular}

.84, y para el de adolescentes, .91. Este índice es sobresaliente en los tres casos (cuadro 1).

Por medio del análisis factorial con componentes principales, valores eigen superiores a uno y rotación varimax, la varianza de $49.7 \%$ se explicó en seis factores para la muestra total (cuadros 1, 2 y 3); de $57.5 \%$ en ocho factores para el grupo de escolares y de $56.5 \%$ en seis factores para el grupo de adolescentes (cuadro 1).

La confiabilidad fue obtenida por dos métodos: 0.88 (alfa de Cronbach) y 0.84 (mitades partidas) para la muestra total; en el grupo de escolares 0.85 (alfa de Cronbach) y 0.80 (mitades partidas), mientras que fue 0.91 (alfa de Cronbach) y 0.87 (mitades partidas) para el grupo de adolescentes (cuadro 1).

Se realizó un análisis de los antónimos para determinar el valor que cada uno aportaba a la confiabilidad total, encontrándose muy buena consistencia interna en la escala (cuadro 4). Asimismo, se intentó hacer un análisis factorial con una solución varimax obligada a tres factores (los encontrados por Barraca y López-Yarto); ${ }^{1}$ sin embargo, la confiabilidad alfa de Cronbach en el tercer factor cae a 0.68 .

Se obtuvieron los percentiles por medio de los cuales puede obtenerse la satisfacción familiar de escolares y adolescentes (cuadro 5).

En el cuadro 6 se aprecia el grado de satisfacción familiar de los sujetos que participaron en este estudio. La mediana de la puntuación obtenida por los escolares fue 140 (satisfacción familiar media); mientras que la de los adolescentes fue 134 (satisfacción familiar media).

Cuadro 6. Interpretación de la Satisfacción familiar (SF) de escolares $(n=237)$ y adolescentes $(n=239)$

\begin{tabular}{lccccc}
\hline Interpretación & \multicolumn{2}{c}{ Escolares } & & \multicolumn{2}{c}{ Adolescentes } \\
\cline { 2 - 3 } \cline { 5 - 6 } SF & $\mathrm{fr}$ & $\%$ & & $\mathrm{fr}$ & $\%$ \\
\hline Muy baja & 48 & 20.4 & & 48 & 20.0 \\
Baja & 25 & 10.5 & 25 & 10.5 \\
Media & 75 & 31.6 & 76 & 31.8 \\
Alta & 24 & 10.1 & 20 & 8.4 \\
Muy alta & 65 & 27.4 & 70 & 29.3 \\
\hline
\end{tabular}

SF Satisfacción familiar. Fr Frecuencia. $\%$ Porcentaje. 


\section{DISCUSIÓN}

Evaluar el tipo de interacción (satisfactoria o no) que establecen los escolares y adolescentes es útil ya que son individuos que se encuentran en formación y desarrollo de sus habilidades emocionales y sociales.

En la versión original española ${ }^{2}$ se encontraron diferencias estadísticamente significativas en la forma de percibir la satisfacción familiar entre hombres y mujeres; sin embargo, en nuestra muestra no fue así, debido quizá a los usos y costumbres en el lenguaje y a que el grupo etario es diferente al considerado en el estudio español.

Considerando el centil 50 del cuestionario como punto de corte para indicar una vivencia satisfactoria o insatisfactoria de la propia familia, se explica el hecho de que los escolares reportaron tener mayor satisfacción familiar que los adolescentes (140 vs. 134 puntos, respectivamente), ya que los primeros, por el periodo de desarrollo en el que se encuentran, tienen una percepción más estable de su familia y de lo que viven en ella, que los adolescentes que se encuentran en un periodo de relativa insatisfacción consigo mismos y con su grupo familiar. Asimismo, esta diferencia estadísticamente significativa $(p=.002)$, indica que el instrumento tiene la capacidad de discriminar entre escolares y adolescentes con respecto a la satisfacción que tienen de su familia.

El análisis factorial es el método más adecuado para comprobar la validez de constructo de un instrumento. ${ }^{1}$ Barraca y López-Yarto describen para la ESFA una varianza acumulada de $70.8 \%$ explicada en tres factores con valores eigen mayores a uno. En este estudio, la varianza bajó hasta $49.7 \%$ y se explicó en seis factores, debido posiblemente a las dificultades tanto de los escolares como de los adolescentes para entender el significado de ciertos antónimos. Sin embargo, si se hace un análisis por separado de escolares y de adolescentes, se puede observar mayor estabilidad y consistencia interna en el grupo de adolescentes $(\alpha=.91)$ que en el de escolares $(\alpha=.86)$.

Una de las principales limitaciones del estudio está dada porque la muestra fue obtenida de una sola zona geográfica de la Ciudad de México con sujetos cuya condición socioeconómica era media-baja, lo que posiblemente determinó la dificultad para entender el significado de ciertos antónimos. Se sugiere tener precaución con el antónimo número seis (sosegado/a-desasosegado/a), ya que, al momento de la aplicación de la escala, más de $90 \%$ de los sujetos manifestaron no entenderlo. Asimismo, se sugiere modificar cinco antónimos por sinónimos para la clara comprensión de la escala por estos grupos etarios. Los cambios se sugieren en el antónimo 3 (jovial por animado/a), 10 (cohibido/a por limitado/a), 14 (atosigado/a por molestado/a), 17 (marginado/a por apartado/a) y 22 (agobiado/a por angustiado/a).

Se puede concluir que la Escala de Satisfacción Familiar por Adjetivos (ESFA) posee muy buena consistencia inter- na, confiabilidad, validez de constructo, validez de contenido y validez discriminante, y que puede ser utilizada en escolares y adolescentes mexicanos.

\section{AGRADECIMIENTOS}

Este trabajo fue realizado mediante la beca 33745 del Consejo Nacional de Ciencia y Tecnología (CONACyT). Es parte del proyecto doctoral titulado "Calidad de vida, ansiedad y depresión en escolares y adolescentes con enfermedades crónicas" (Protocolo HIM/2010/068) en el Programa de Maestría y Doctorado en Ciencias Médicas, Odontológicas de la Salud, de la Universidad Nacional Autónoma de México.

\section{REFERENCIAS}

1. Guijarro EA. Estructura y funcionamiento familiar de niños con cáncer desde la perspectiva de las madres. Tesis doctoral. Universidad de la Laguna. España 2010. ISBN: 978-84-7756-971-8.

2. Barraca MJ, López-Yarto EL. ESFA. Escala de satisfacción familiar por adjetivos. Madrid, España: Segunda edición. TEA, Publicaciones de Psicología Aplicada; 1999.

3. Rollan GC, García-Bermejo M, Villarrubia I. Relación entre la satisfacción familiar, el bienestar psicológico y el sentido de la vida. www. cesdonbosco.com. Accesado en abril de 2011.

4. Martínez-Pampliega A, Iraurrgi I, Galíndez E, Sanz M. Family adaptability and cohesion evaluation scale (FACES): Desarrollo de una versión de 20 ítems en español. International J Clinical Health Psychology 2006;6(2):317-338.

5. Luengo RT, Román SJM. Estructura familiar y satisfacción parental: propuestas para la intervención. Acciones e investigaciones sociales, ISSN 1132-192X $\mathrm{N}^{\circ}$ extra 1, 2006:455.

6. Berge JM, Holm KE. Boundary ambiguity in parents with chronically ill children: Integrating theory and research. Family Relations 2007;56(2):123-134.

7. Christians LA. Fathers of chronically ill children: Assessment and perceived effectiveness of their coping methods. ProQuest Information and Learning Company. 2004, UMI Microfilm: 1421655.

8. Vulic-Prtoric A, Macuka I. Family and coping factors in the differentiation of childhood anxiety and depression. Psychology Psychotherapy 2006;79:199-214.

9. Grossman LK, Rich LN, Michelson S, Hagerty G. Managed care of children with special health care needs: The ABC program. Clinical Pediatrics 1999;38(3):153-160.

10. Mahat G, Scoloveno MA, Barnette DC. Written educational materials for families of chronically ill children. J American Academy Nurse Practitioners 2007;19(9):471-476.

11. Mikulic IM. Construcción y adaptación de pruebas psicológicas. Universidad de Buenos Aires. Facultad de Psicología. www.psi.uba.ar/ academica/carrerasdegrado/.../059.../f2.pdf. Recuperado 29 mayo 2011.

12. Malo-Salvatierra DA. La medición en psicología como herramienta y como reflexión ética en el ejercicio del psicólogo. Psicogente 2008;11(19):46-51.

13. Sánchez R, Echeverry J. Validación de escalas de medición en salud. Rev Salud Pública 2004;6(3):302-318.

14. Fernández AL, Marino JC, Alderete AM. Estandarización y validez conceptual del test del trazo en una muestra de adultos argentinos. Revista Neurológica Argentina 2002;27:83-88.

15. Botía SML, Orozco PLH. Validación preliminar de una batería de solución de problemas. Cognición Revista Científica de FLEAD. http:// cognicion.net. Recuperado 29 mayo 2011. 
16. Isasi BX, Balluerka LN, Gorostiaga MA. La utilización de instrumentos de medida en situaciones de contacto de lenguas y de culturas: una reflexión metodológica. Psicothema 2002;12(sup 2):305-310.

17. Nunnally JC, Bernstein IH. Teoría psicométrica. México: Tercera edición. Mc Graw Hill; 1994: pp. 37-43, 92-127, 277-378.

18. Aliaga TJ. Psicometría: tests psicométricos, confiabilidad y validez. En: www.unmsm.edu.pe/psicologia/.../05Libro EAPAliaga.pdf. Recuperado 29 mayo 2011.
19. Villagómez DG. Adaptación mediante análisis factorial del (CTI) para población ecuatoriana de la Ciudad de Quito. Pontificia Universidad Católica del Ecuador; Facultad de Psicología; 2007.

20. Espinoza MJC. Validación y estandarización de instrumentos. Universidad Nacional de Colombia. Colombia: Convenio Interadministrativo de Cooperación Académico-Científico; 2008; 29: 1-16.

Artículo sin conflicto de intereses 